\title{
Erratum: Group-theoretical classification of superconducting states of strontium ruthenate [Phys. Rev. B 100, 214507 (2019)]
}

\author{
S.-O. Kaba and D. Sénéchal $\odot$
}

(Received 11 April 2020; revised manuscript received 18 April 2020; published 7 May 2020)

DOI: 10.1103/PhysRevB.101.209901

In our paper, the automated generation of pairing functions in the presence of spin-orbit coupling was erroneous in the case of the $u$-type representations of $D_{4 h}$ and $D_{2 h}$ due to a programming error. This invalidates much of Table $\mathrm{V}$ of our paper as well as the rightmost sections of Figs. 3 and 6 and some comments made in the discussion section. Corrected versions are included in this Erratum. The general theory and procedure used to obtain the pairing functions are unchanged as is the discussion surrounding pure singlet and pure triplet states. In our paper, the pairing functions belonging to each representation of the point group of $D_{4 h}$ (and $D_{2 h}$ in the presence of uniaxial stress) were generated automatically by a program written in Python. A small error in that program resulted in erroneous results in the presence of spin-orbit coupling that affected the odd representations of $D_{4 h}\left(A_{1 u}, A_{2 u}, B_{1 u}, B_{2 u}\right.$, and $\left.E_{u}\right)$ and those of $D_{2 h}\left(A_{u}, B_{1 u}, B_{2 u}\right.$, and $\left.B_{3 u}\right)$. An error also affected the shape of nodal lines in all representations in the presence of spin-orbit coupling.

This affects most of Table V, as well as the two rightmost columns of Figs. 3 and 6. A corrected Table V is included on the next page. In the paper, we chose to include only the simplest pairing functions (the lowest-degree polynomials) for each type of intraband or interband pairing. Even with this restriction, the number of possible pairing functions is too large to fit in one page in the spin-orbit case; therefore, we omitted the $E_{g}$ and $E_{u}$ representations from the corrected table.

The correction to the rightmost two columns of Fig. 3, representing the generic nodal lines in the presence of spin-orbit coupling, is shown on this Erratum's Fig. 1. It is obtained by adding all the pairing functions of a given representation in order to approximate what we could call a generic pairing function, allowed by symmetry. However, such as in the original paper, it must be kept in mind that small variations in the nodal lines may occur if the amplitudes of the different components are allowed to vary relative to one another. We sampled a few variations, and they are, indeed, very small. Figure 2 of the Erratum likewise corrects the last two columns of Fig. 6 of the paper in the case of uniaxial stress.

These changes force us to correct some of the comments of the discussion section:

(1) It is no longer true that the only representations without nodes are $A_{1 g}$ and $E_{u}(1, i)$. In the presence of spin-orbit coupling, one must also add $A_{1 u}, A_{2 u}$, and $B_{2 u}$ to this list.

(2) Likewise, in the presence of uniaxial stress, the representations $A_{g}, A_{u}$, and $B_{1 u}$ are nodeless.

(3) Only the $E_{g}(1,0)$ and $E_{g}(1,1)$ representations have both vertical and horizontal nodal lines, and both of these are even under inversion. This invalidates a statement made in Sec. IV D of the paper in that respect, saying that this was a property of representations $A_{1 u}$ and $B_{1 u}$. The other statement made about these two representations in the same section is also incorrect.

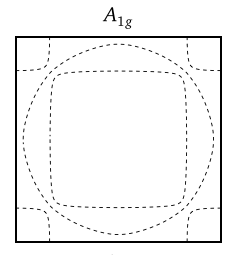

$A_{1 u}$
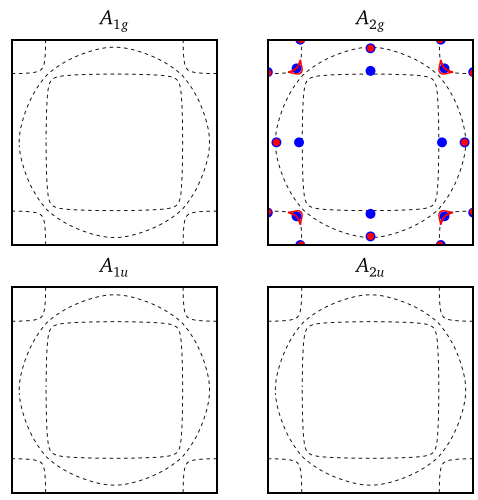

$A_{2 u}$
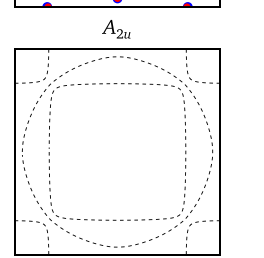

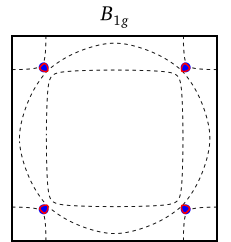

$B_{1 u}$

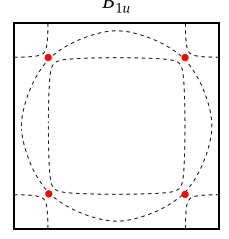

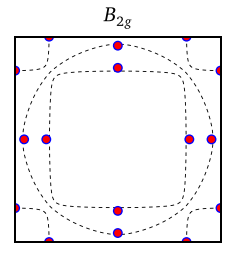

$B_{2 u}$

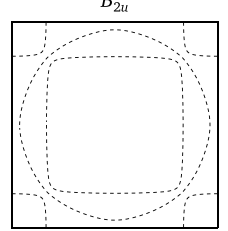

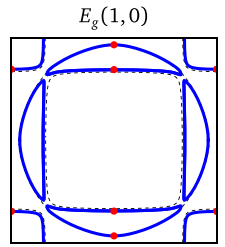

$E_{u}(1,0)$

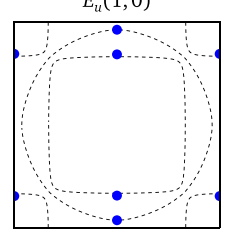

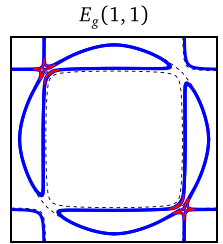

$E_{u}(1,1)$

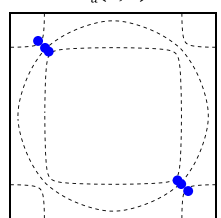

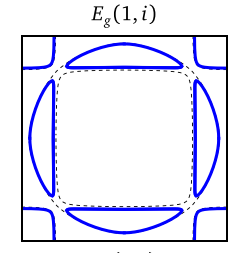

$E_{u}(1, i)$

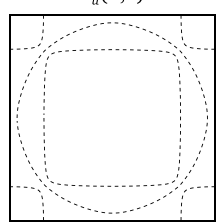

FIG. 1. Averaged or typical nodes associated with the different irreps of $D_{4 h}$ for $k_{z}=0$ (blue) and $k_{z}=\pi / 2$ (red), in the presence of spin-orbit coupling. Each panel covers the full Brillouin zone from $(-\pi,-\pi)$ to $(\pi, \pi)$, and the representation label is indicated on top. This replaces the rightmost two columns of Fig. 3 of the paper. 
TABLE V. List of pairing functions with spin-orbit coupling. For conciseness, the $E_{g}$ and $E_{u}$ representations are not shown. See Table III of the paper for an explanation of the symbols.

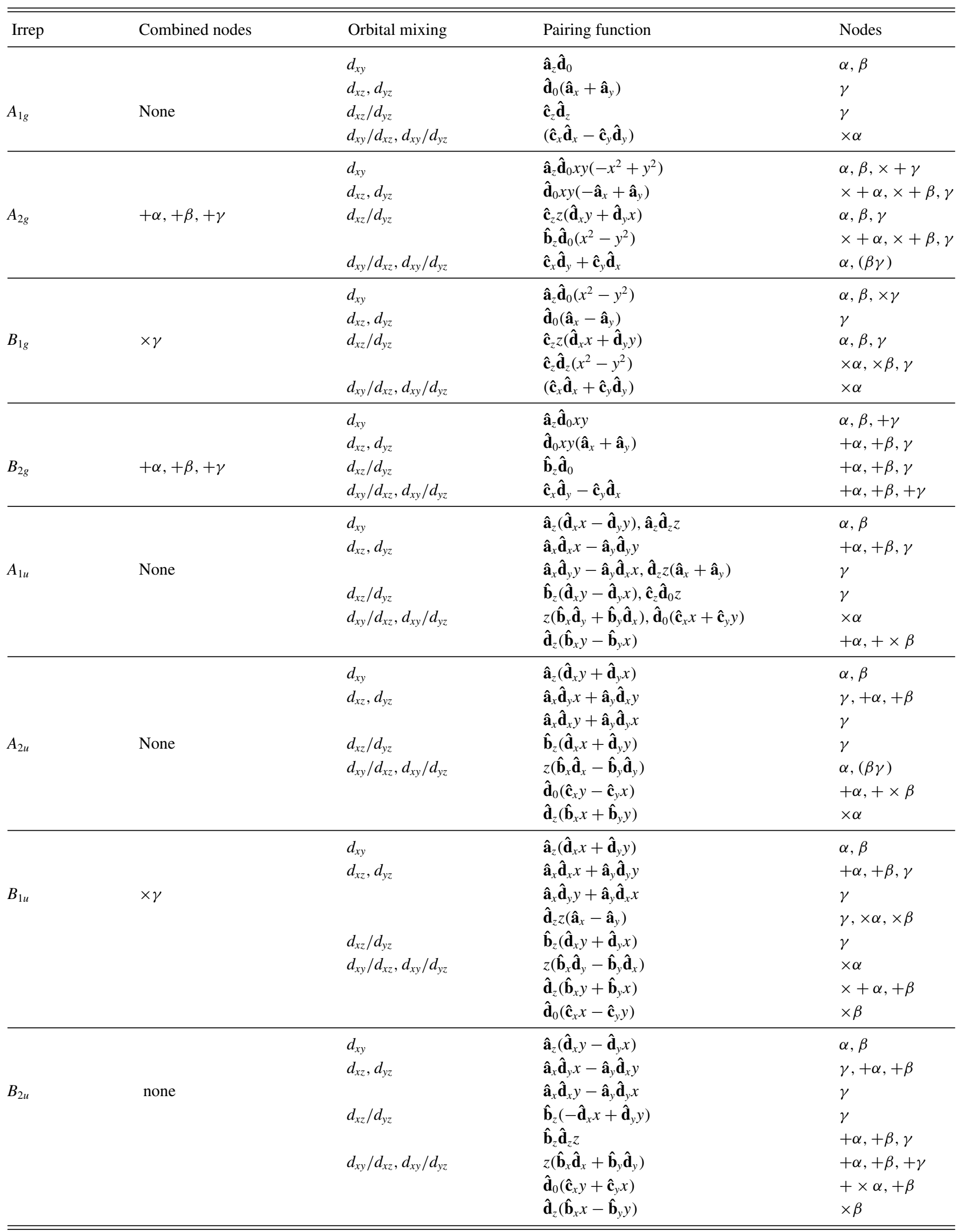



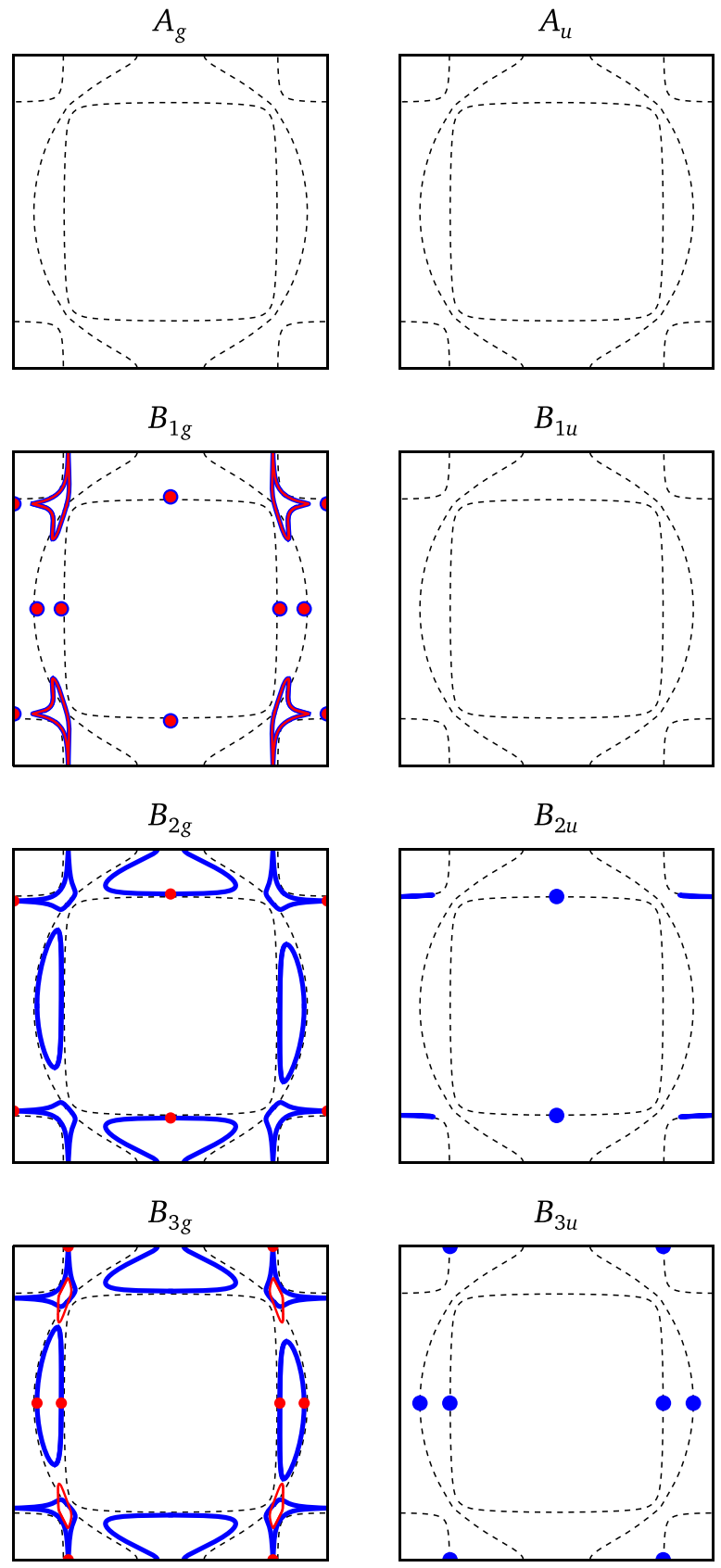

FIG. 2. Averaged or typical nodes associated with the different irreducible representations of $D_{2 h}$ for $k_{z}=0$ (blue) and $k_{z}=\pi / 2$ (red) in the presence of spin-orbit coupling. Each panel covers the full Brillouin zone from $(-\pi,-\pi)$ to $(\pi, \pi)$, and the representation label is indicated on top. This replaces the rightmost two columns of Fig. 6 of the paper. 